\title{
Deterministic and Probabilistic Assessment of the Impact of the Electrical Vehicles on the Power Grid
}

\author{
E. Valsera-Naranjo ${ }^{1}$, A. Sumper ${ }^{1,2}$, P. Lloret-Gallego ${ }^{1}$, R. Villafáfila-Robles ${ }^{1}$, A. Sudrià-Andreu ${ }^{1,2}$ \\ ${ }^{1}$ Centre d'Innovació Tecnològica en \\ Convertidors Estàtics i Accionaments(CITCEA-UPC), Departament d'Enginyeria Elèctrica, \\ Universitat Politècnica de Catalunya. \\ EU d'Enginyeria Tècnica Industrial de Barcelona, \\ Comte d'Urgell, 187; 08036 Barcelona, Spain. http://www.citcea.upc.edu \\ ${ }^{2}$ IREC Catalonia Institute for Energy Research \\ Josep Pla, B2, Pl. Baixa. 08019 Barcelona, Spain \\ http://www.irec.cat \\ eduard.valsera@citcea.upc.edu,sumper@citcea.upc.edu, lloret@citcea.upc.edu, \\ roberto.villafafila@citcea.upc.edu, sudria@citcea.upc.edu
}

\begin{abstract}
This paper analyzes the impact of the charge of EVs (Electrical Vehicles) on a power grid. In order to simulate the behavior of the EV charging on the grid a model of its battery has been found (EV's charging curve). As the way of the EV is charged from the grid affects critically to the voltage levels and to the saturation of the lines two modalities of charge are presented: not-controlled charge and controlled charge. Finally, the simulations have been performed from two points of view: deterministic analysis and probabilistic analysis.
\end{abstract}

\section{INTRODUCTION}

The recent awareness about fossil fuels and the environment has arisen more sustainable alternatives regarding means of transport. Consequently, hybrid vehicles and pure electrical vehicle have become the main alternatives for green transportation. This new trend has caused market activation and it is expected that hybrid and electrical vehicles will constitute the majority in private transport.

Moreover, one kind of hybrid vehicles, the plug-in electric vehicles (PHEV), will not only charge their batteries, but PHEV will also be able to inject power to the network when required, as the electrical vehicles (EV) do. This fact suggests that EV penetration will affect current power system performance. Then, it is necessary to study some scenarios of penetration of such vehicles into the electrical network in order to maintain security and quality of power supply within standard limits.

On one hand, in order to analyze the impact of EVs integration into the power grid, aspects related to storage technologies (namely batteries) and the charging process of such storage devices have to be studied. In order to analyze the impact of EV on the power grid, load flow calculations in a standard urban network are performed following. To do that, different penetrations of EV into the network are considered. The results of simulations are shown and commented lately.

On the other hand, an European standard which defines the type of connector to be used by EVs is needed. Until a new standard gets develop, it is proposed to use a SCHUKO (CEE $7 / 4$ ) connector type for currents up to 16A. In addition, to slow charging, the output values of the charging station should be up to $16 \mathrm{~A}$ per plug, $230 \mathrm{~V} \pm 10 \%$, and $50 \mathrm{~Hz} \pm 1 \%$ [1].

\section{Electrical Vehicle}

In order to analyze the impact of the EVs into the grid, a model of its charging curve is needed. From all the existing models of EV, the charging curve chosen is from the Mitsubishi I-MIEV [2]. This vehicle has an autonomy of $160 \mathrm{~km}$ and its batteries are made of Li-ion (50Ah 16kWh 330V) [2]; Fig. 1 shows the charging curve of the EV when its capacity at $20 \%$. The battery is charging at full power during 2 hours and then decreases its charging power exponentially since the hour 4 ; at this time it is considered that the battery is completely charged.

The way of how EVs are charged from the grid has a critical influence on its impact on the voltage levels and on the saturation of the lines. Considering this fact, two modalities of charge are presented: not-controlled charge and controlled charge. In the not-controlled charge EVs start the charge as they park. In the controlled charge, EVs only can charge during a determined period of time of the day (low-load period). In both modalities of charge, the number of EVs charging 


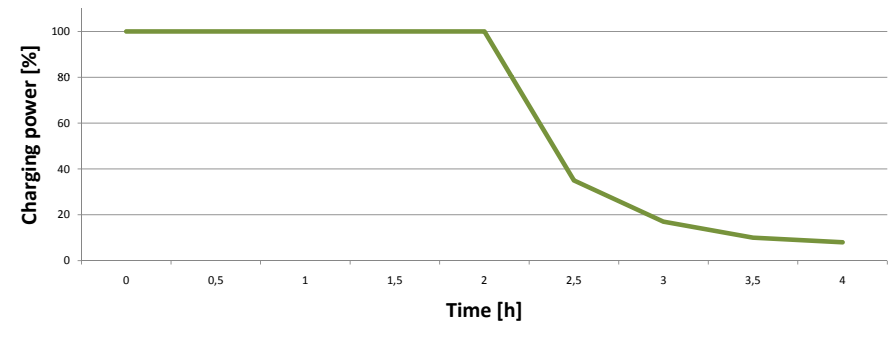

Fig. 1. Charging curve of the EV

is defined establishing different scenarios of EV penetration over the vehicles acceding to the city (Fig.2). The curve of the movement of the vehicles has been obtained from an estimation of the curve of Barcelona [3] and has been shifted two hours earlier in order have a better representation of the Denmark's vehicle movement (in this estimation, population has also been considered).

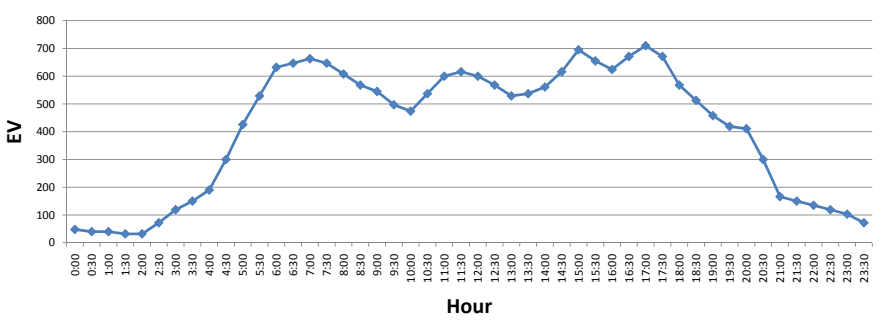

Fig. 2. Movement of vehicles

During the charging process of the EV, will be charging simultaneously EV with different states of charge. Therefore, a model is needed to take this fact into account in the steadystate simulations. Eq. 1 models the superposition of the demand caused by the EV in different charging states, where $R$ is penetration of EV, Pev is the maximum power which a car can charge, $i$ is the counter associated to the actual hour, $j$ is the counter associated to the previous hour, $\Delta v a_{k, k-1}$ is the increase of $\mathrm{EV}$ between the hour $i$ and the hour $j$ and $C e v_{k}$ is the charging state of the EV (Fig.1).

$$
P H_{i}=R \cdot P e v \cdot \sum_{k=1}^{i}\left(\Delta v a_{k, k-1} \cdot C_{e v}\right)
$$

Applying this model to the vehicle movement curve, the demand curves for the both charging modalities can be obtained (Fig.3)

\section{POWER GRID}

The scenario of the simulations is a part of the Danish sample grid (Fig.4) [4]. This grid, of $400 \mathrm{MW}$ of short-circuit power, has 3 wind turbine generation units of $630 \mathrm{~kW}$ at busbars B013, B015 and B017. In addition, there are three combined cycle units of $3 \mathrm{MW}$ each one at the busbar B005, but for purposes of the study has remained disconnected. Loads are at the busbars B005, B010, B011 and B012. Due to simplify the interpretation of the simulations and the results, EVs have been separated from the other consumptions.

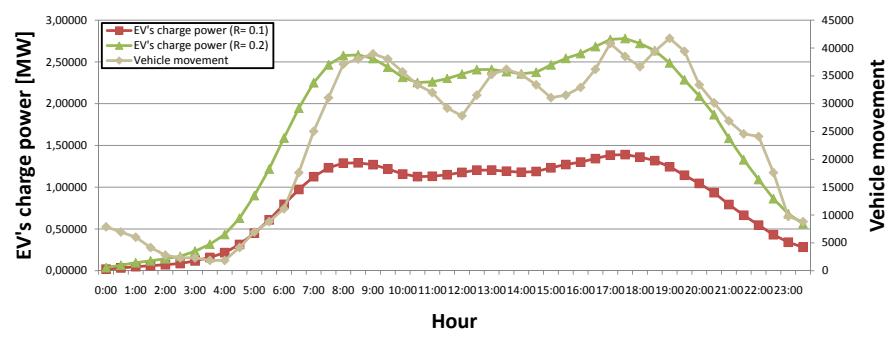

(a) Not-controlled charge

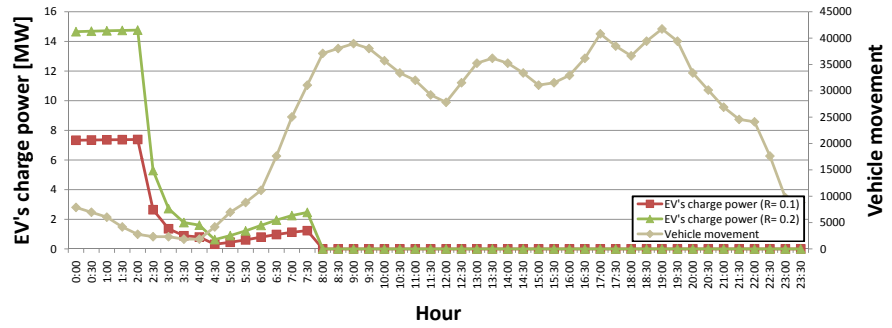

(b) Controlled charge

Fig. 3. EV's power demand for the not-controlled and controlled charge modality

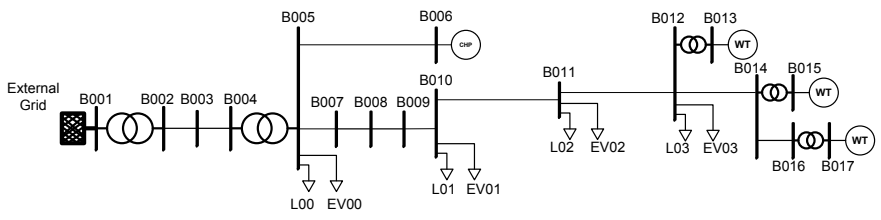

Fig. 4. Danish power grid

In order to study the most critical situation the Winter(week) case has been chosen from all the possible situations of electrical demand because it has the highest demand in every hour (Fig.5). Therefore, the wind power generation curve is from this period. Fig. 6 depicts a representative daily generation for the three wind power generation units.

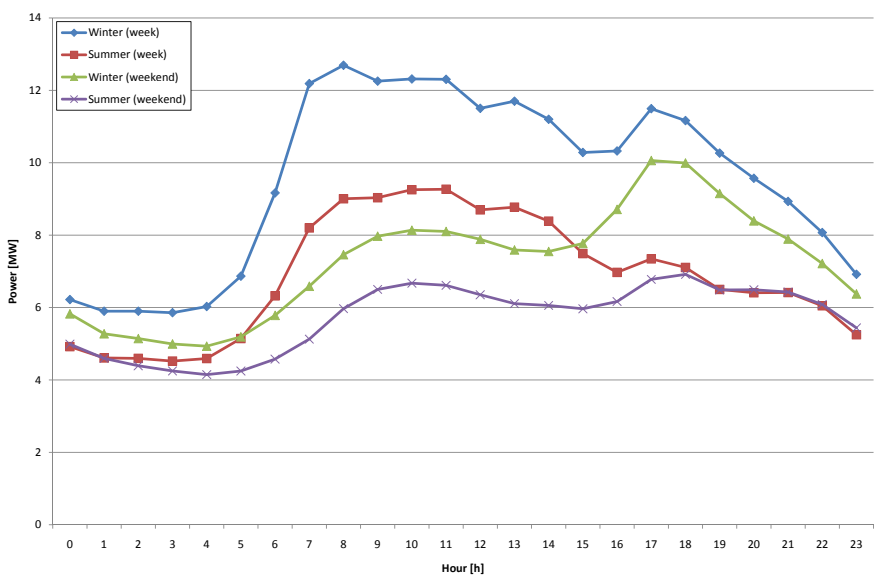

Fig. 5. Electrical demand

In the deterministic and in the probabilistic analysis the voltage results presented are from two busbar. The first busbar is B005 and it has been chosen because L00 is always the load with the highest power. The second busbar chosen is B014 in order to have the behavior of a closer busbar to a wind power 


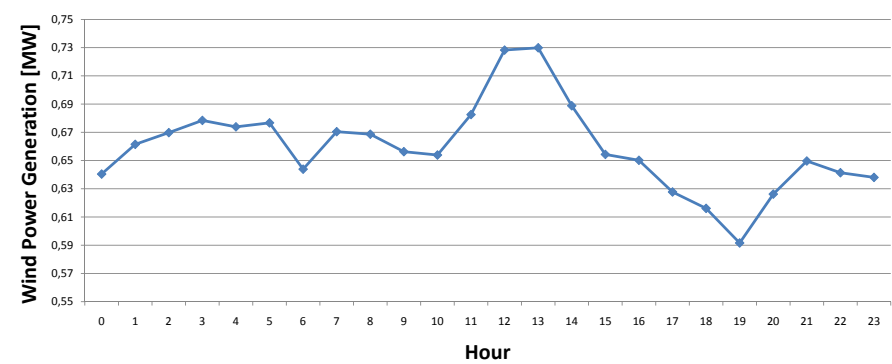

Fig. 6. Wind power generation

generation unit.

\section{Deterministic AnAlysis}

In this section, the result from executing a deterministic analysis are shown. In this kind of analysis all loads are previously determined. The results from the two typologies of charge for the EV are presented for the different penetrations of EV.

\section{A. Not-controlled charge}

Fig.7 and Fig.8 show respectively the voltage levels for busbar B005 and B014 for the case of not-controlled charge.

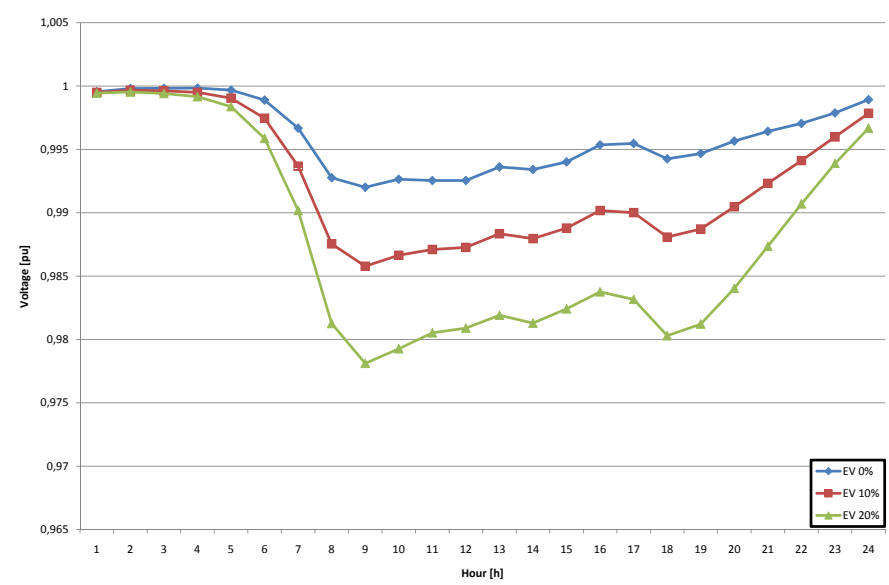

Fig. 7. Voltage levels for the busbar B005 for the different penetrations of $\mathrm{EV}$ in the case of not-controlled charge for the deterministic analysis

\section{B. Controlled charge}

Fig.9 and Fig.10 show respectively the voltage levels for busbar B005 and B014 for the case of controlled charge.

\section{Probabilistic Analysis}

From the compiled data during the week days of the winter season a mean and an standard deviation have been found for each hour of the day. In order to perform a probabilistic analysis 50 load flows for each hour have been executed generating random powers with the mean and the standard deviation founded previously.

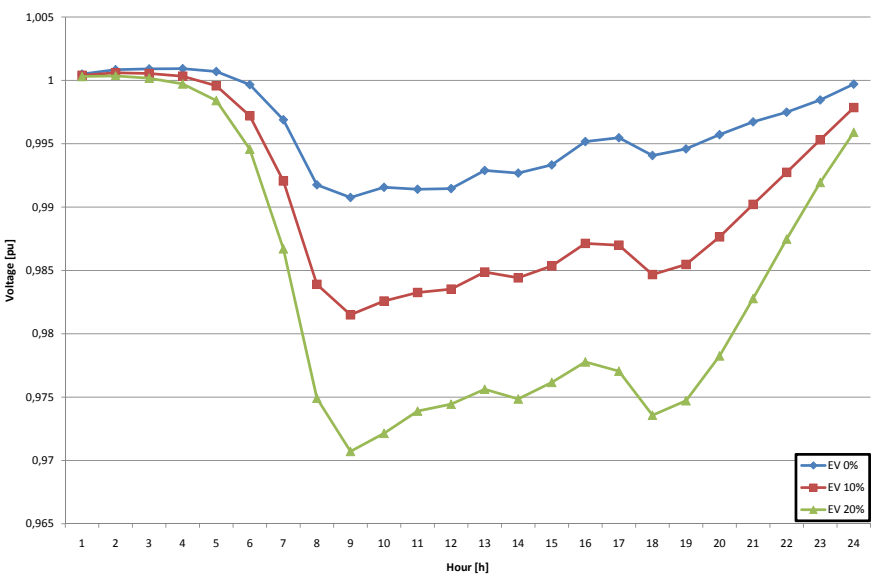

Fig. 8. Voltage levels for the busbar B005 for the different penetrations of $\mathrm{EV}$ in the case of not-controlled charge for the deterministic analysis

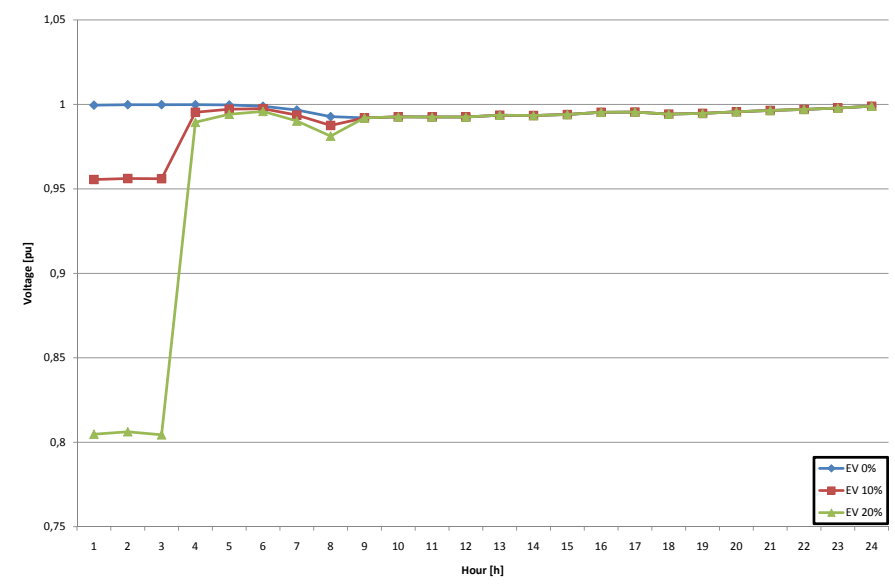

Fig. 9. Voltage levels for the busbar B005 for the different penetrations of $\mathrm{EV}$ in the case of controlled charge for the deterministic analysis

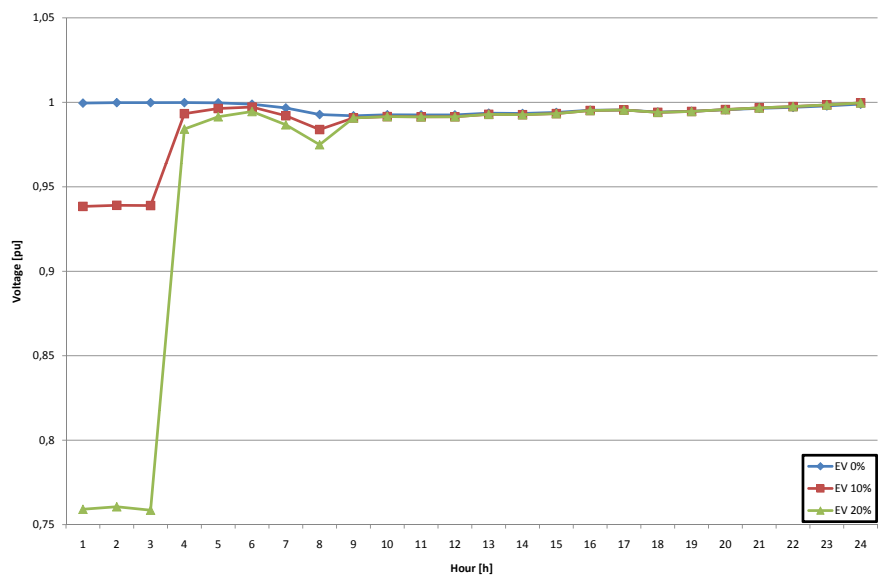

Fig. 10. Voltage levels for the busbar B005 for the different penetrations of $\mathrm{EV}$ in the case of controlled charge for the deterministic analysis 


\section{A. Not-controlled charge}

Fig.11 and Fig.12 show mean voltage levels for the different penetrations of $\mathrm{EV}$ in the case of not-controlled charge. In both figures are the calculated mean voltage levels from the probabilistic analysis and the mean from the deterministic analysis. In order to have a most detailed view of the critical busbar, in this case B014, Fig.13 show the the range of voltage level that can be reached at this busbar.

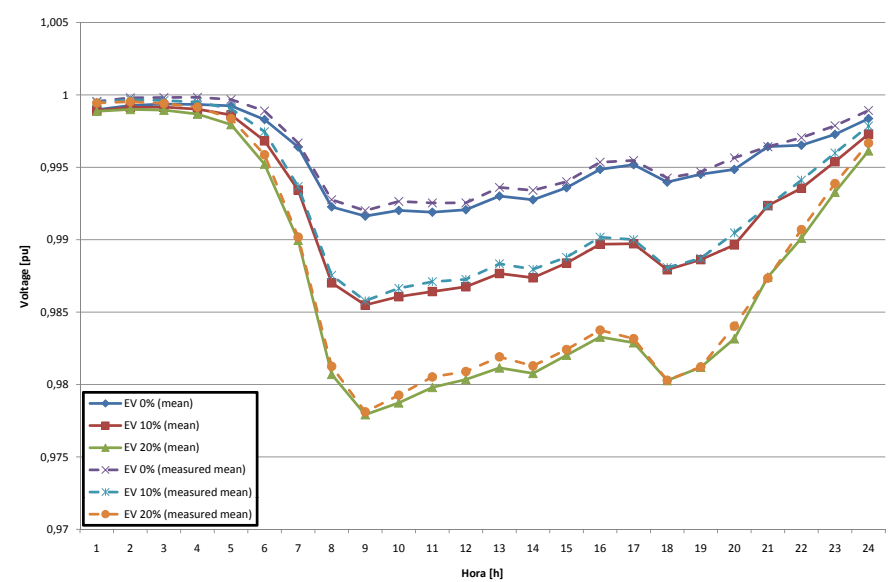

Fig. 11. Voltage levels for the busbar B005 for the different penetrations of $\mathrm{EV}$ in the case of not-controlled charge

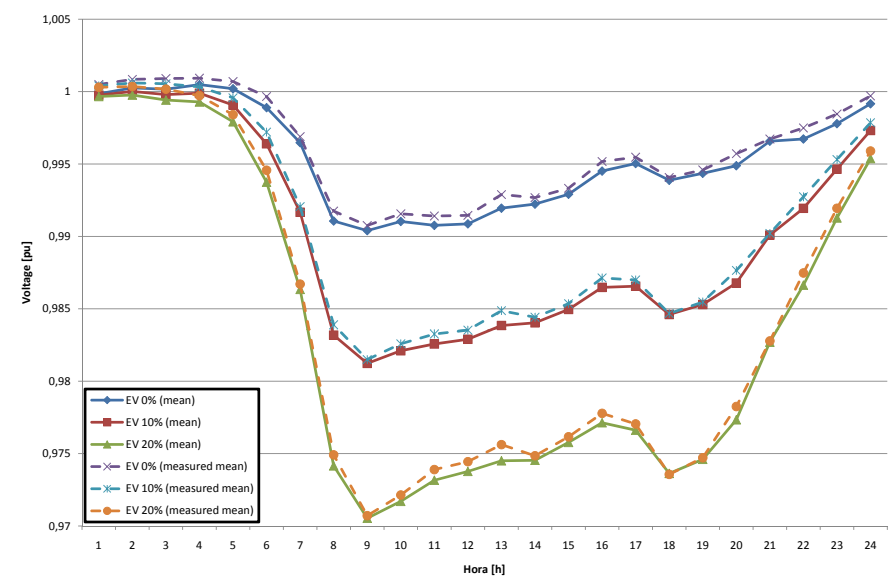

Fig. 12. Voltage levels for the busbar B014 for the different penetrations of $\mathrm{EV}$ in the case of not-controlled charge

\section{B. Controlled charge}

Fig.14 and Fig.15 show mean voltage levels for the different penetrations of $\mathrm{EV}$ in the case of controlled charge. As happens in the not-controlled charge, the B014 is the busbar with the lowest voltage levels, Fig.16 show the the range of voltage level that can be reached at this busbar.

\section{CONCLUSION}

The objective of the paper is to analyze the impact of charging EVs from a Danish grid. Therefore, different penetrations of EV and charge modalities are proposed. In order to cover

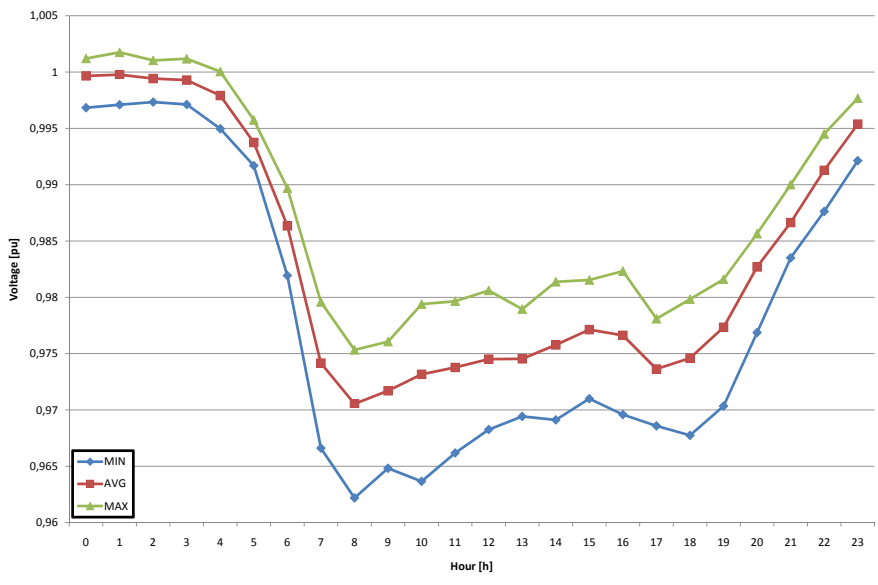

Fig. 13. Voltage levels for the busbar B005 for the different penetrations of $\mathrm{EV}$ in the case of not-controlled charge

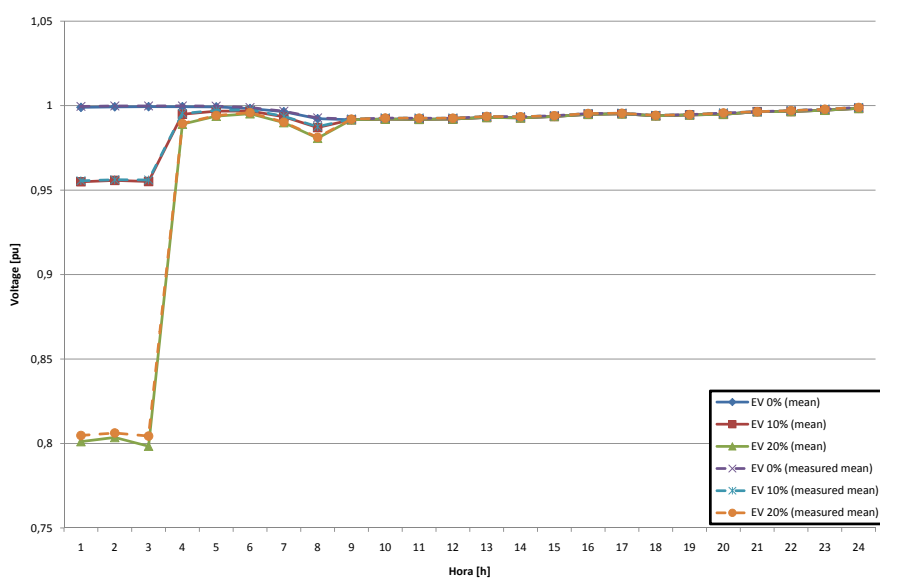

Fig. 14. Voltage levels for the busbar B005 for the different penetrations of $\mathrm{EV}$ in the case of controlled charge

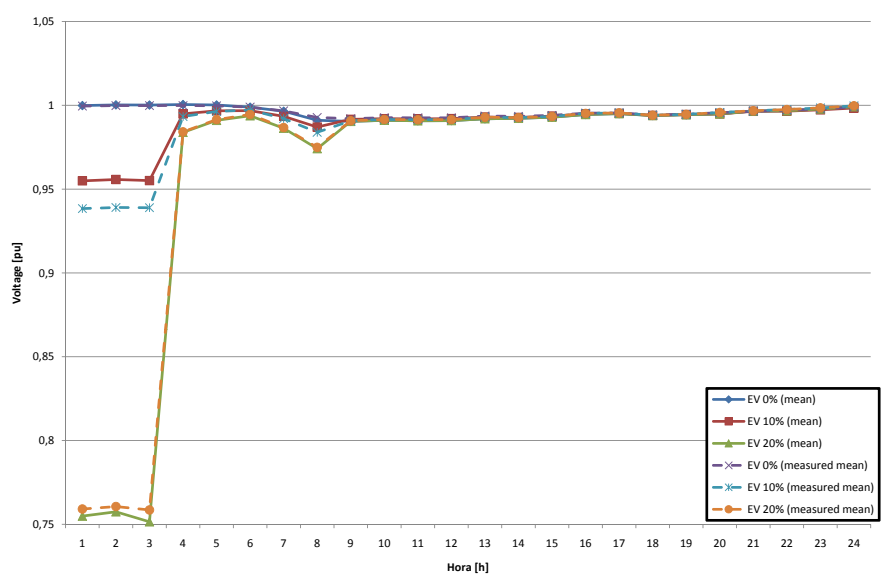

Fig. 15. Voltage levels for the busbar B005 for the different penetrations of $\mathrm{EV}$ in the case of not-controlled charge 


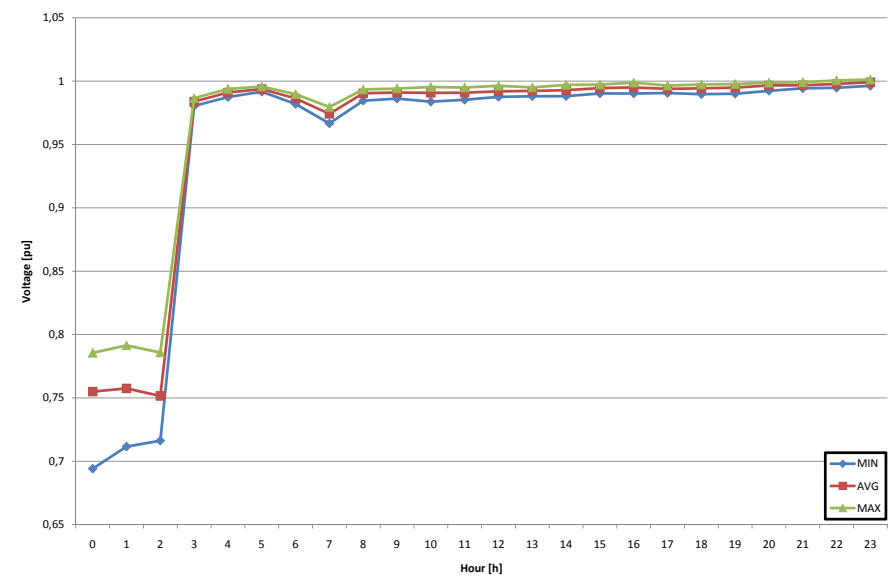

Fig. 16. Voltage levels for the busbar B005 for the different penetrations of $\mathrm{EV}$ in the case of not-controlled charge

the maximum situations two analysis have been performed: deterministic analysis and probabilistic analysis.

On one hand, results from the simulations show that notcontrolled charge amplifies the demand of the line at the hours of the higher electrical demands. On the other hand, simulations show that with the controlled charge a high penetration of EVs can be charged without the need of investments in the power grid infrastructure.

\section{REFERENCES}

[1] Valsera-Naranjo Sumper Lloret-Gallego Villafáfila-Robles, SudriàAndreu. Electrical vehicles: state of art and issues for their connection to the network. Electrical Power Quality and Utilisation, 2009. 1

[2] http://www.mitsubishi-motors.com/special/ev/index.html. 1

[3] Dades Bàsiques 2008 v.02. Ajuntament de Barcelona. Direcció de Serveis de Mobilitat., 2008. 2

[4] Roberto Villafáfila-Robles. Probabilistic modeling in normal operation and the control of distribution systems with renewable source based DG units. PhD thesis, Universitat Politècnica de Catalunya, 2009. 2 